\section{Preliminary results of allograft use from the South African skin bank}

To the Editor: In 2013, Rogers et al. ${ }^{[1]}$ highlighted the desperate need for a skin bank in this country. Earlier this year, Allorto et al. ${ }^{\left[{ }^{[2]}\right.}$ in a letter to this journal, reported the establishment of South Africa (SA)'s first skin bank, and advocated for increased donation, as well medical and lay education to increase tissue donation to meet the enormous need.

The primary motivation for this initiative is to have a ready supply of deceased donor (cadaver) allograft for use as a biological temporary skin substitute. In the context of major burn injury, allograft is used as a temporary wound cover after excision of deep partial thickness and full thickness burns prior to autografting, or as physical protection and a scaffold over widely meshed autografts. Allograft is the best possible test of the capacity for a wound bed to accommodate subsequent autografting, especially in the context of a major burn with limited donor sites, but is also invaluable following the management of wound infection, necrosis or other conditions that may compromise take. Excellent results have also been reported in the context of exfoliative disorders of the skin or as part of reconstructive surgeries to create a dermal scaffold (referred to as alloderm or allodermis).

Burns exceeding $40 \%$ of the total body surface area (TBSA) have inadequate donor skin available to cover the excised burn eschar. Temporary alternatives to autografts include synthetic skin substitutes or deceased donor allograft. Allografts can be sourced from organ donor patients, hospital or state mortuaries, private institutions or (rarely) living donors. Using restrictive guidelines for its use, at least 25 patients per month with major burns would benefit from allograft use as part of their burn care in SA. The establishment of a national skin bank attached to the Tshwane University of Technology ensures the availability of a legal, secure and readily available source of quality allograft. The bank was officially opened in April 2016 and is supported by the Department of Health and the SA Tissue Bank (SAtiBA).

Similar to the European skin bank, allograft preservation is through an inexpensive process of glycerolisation. Once deglycerolised, the product functions as a valuable temporary skin cover, undergoing the phases of graft take not unlike autograft (plasmatic imbibition, inosculation and neovascularisation). Tissue compatibility between donor and recipient is not required, although rejection is inevitable if skin is left on for more than 2 weeks, resulting in epidermolysis. The skin is commercially available at $\mathrm{R} 11 / \mathrm{cm}^{2}$ and can be stored for at least 2 years at $4^{\circ} \mathrm{C}$.

We present our preliminary results from eight paediatric patients managed at the Red Cross War Memorial Children's Hospital using glycerolised allografts obtained from the skin bank. The mean age was 3.6 years (range 10 months to 8 years). Seven sustained flame burns and one a deep scald injury. The indications for allograft use in all eight were cover following burn eschar excision and wound preparation, and to enhance epithelialisation after micrografting in five patients. The mean TBSA burn was $49 \%$ (range $11-86 \%$ ). Allografts were placed immediately after early excision, typically on day 3 (range 1 - 9 days). Autograft take following allograft wound preparation was greater than $90 \%$ in all cases. The TBSA grafted with allograft in these eight children was $35.9 \%$; this was a total of $12100 \mathrm{~cm}^{2}$ allograft. All the children survived and were discharged, following shorter average hospitals stays than before.

We used allograft to optimise wound bed preparation after excision, contributing to excellent outcomes after removal of the micrograft sheets on the seventh postoperative day. Having allograft available at both of these critical phases has undoubtedly resulted in shorter hospital lengths of stay (less than 1.5 days per percentage burn), and increased the lethal dose, $50 \%\left(\mathrm{LD}_{50}\right)$ of major burns in this unit to over 70\% TBSA, a statistic unprecedented in low- and middle-income countries. Another paediatric burn unit in SA, Chris Hani Baragwanath Hospital, also started using skin bank allograft recently, where it was applied to four children with burns between 30 and 50\% TBSA as part of temporary skin cover after initial excision.

The skin bank has changed the landscape for the management of major burns in SA.

\section{H Rode, R Martinez}

Red Cross War Memorial Children's Hospital and Division of Paediatric Surgery, Department of Surgery, Faculty of Health Sciences, University of Cape Town, South Africa

heinz.rode@uct.ac.za

\section{A D Rogers}

Ross Tilley Burn Centre, Sunnybrook Health Sciences Centre; and Division of Plastic and Reconstructive Surgery, Faculty of Medicine, University of Toronto, Canada

\section{R Moore}

Chris Hani Baragwanath Hospital Johnson and Johnson Burns Unit, Faculty of Surgery, University of Witwatersrand, Johannesburg, South Africa

\section{N L Allorto}

Edendale Hospital Burn Service, Pietermaritzburg; and Department of Surgery Nelson R Mandela School of Medicine, College of Health Sciences, University of KwaZulu-Natal, Durban, South Africa

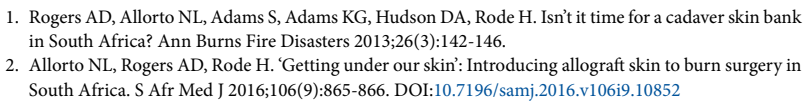

1. Rogers AD, Allorto NL, Adams S, Adams KG, Hudson DA, Rode H. Isn't it time for a cadaver skin bank in South Africa? Ann Burns Fire Disasters 2013:26(3):142-146.

2. Allorto NL, Rogers AD, Rode H. 'Getting under our skin': Introducing allograft skin to burn surgery in South Africa. S Afr Med J 2016;106(9):865-866. DOI:10.7196/samj.2016.v106i9.10852

S Afr Med J 2016:106(11):1059. DOI:10.7196/SAMJ.2016.v106i11.12096 